\title{
A Novel Molecular Mechanism of Severe Congenital Factor XIII Deficiency
}

\author{
Jingjing $\mathrm{Han}^{1}$, Miao Jiang ${ }^{1}$, Jian $\mathrm{Su}^{1}$, Ziqiang $\mathrm{Yu}^{1}$, Xia Bai ${ }^{1}$, Zhaoyue Wang ${ }^{1}$, Changgeng \\ Ruan $^{1}$, Lijun Xia ${ }^{1}$, Yanyan Bai $^{2}$, and Lijuan $\mathrm{Cao}^{1}$ \\ ${ }^{1}$ First Affiliated Hospital of Soochow University \\ ${ }^{2}$ University of Illinois at Chicago
}

July 15,2020

\begin{abstract}
Coagulation factor XIII (FXIII) deficiency is a rare recessive bleeding disorder. Severe patients are caused by a complete absence of FXIIIA protein in plasma, but researches on the molecular mechanisms are limited. This study was to analyze the pathogenic mechanisms of three congenital FXIII deficiency patients. Plasma FXIII activities and antigens were significantly decreased in patients compared to healthy controls by fluorescent assay and western blot. One frameshift mutation and four missense mutations (p.Trp188Cys, p.His374Arg, p.Thr559Pro and p.His717Arg) around the catalytic core were identified. The mRNA levels of four mutants were nearly normal in COS7 cells, and the FXIIIA protein could be detected in cell lysis but not be expressed in the supernatant. Confocal microscopy studies showed clear outlines of cells with both wild FXIII and mutants, but some mutants gathered into clusters neither in the endoplasmic reticulum nor in the Golgi apparatus of cytoplasm, compared to the diffusely-distributed wild FXIII. Considering the above, we concluded that the three cases of FXIII deficiency were caused by p.Trp188Cys, p.His374Arg, p.Thr559Pro and p.His717Arg mutations in FXIIIA gene, which led to the gathering of FXIIIA in cytoplasm and then blocking its transportation into medium.
\end{abstract}

\section{Hosted file}

A Novel Molecular Mechanism of Severe Congenital Factor XIII Deficiency-HUMAN MUTATION.doc available at https://authorea.com/users/342923/articles/469699-a-novel-molecular-mechanismof-severe-congenital-factor-xiii-deficiency

\section{Hosted file}

Table1.docx available at https://authorea.com/users/342923/articles/469699-a-novelmolecular-mechanism-of-severe-congenital-factor-xiii-deficiency

FIGURE 1

A

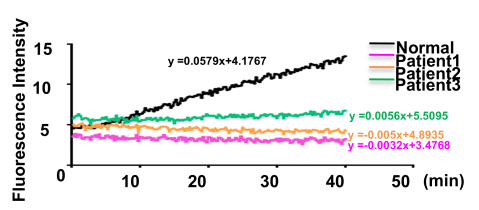

B

Normal Patient $1 \quad$ Patient 2 Patient 3

FXIII-B

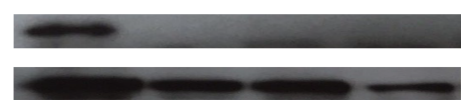


FIGURE 2

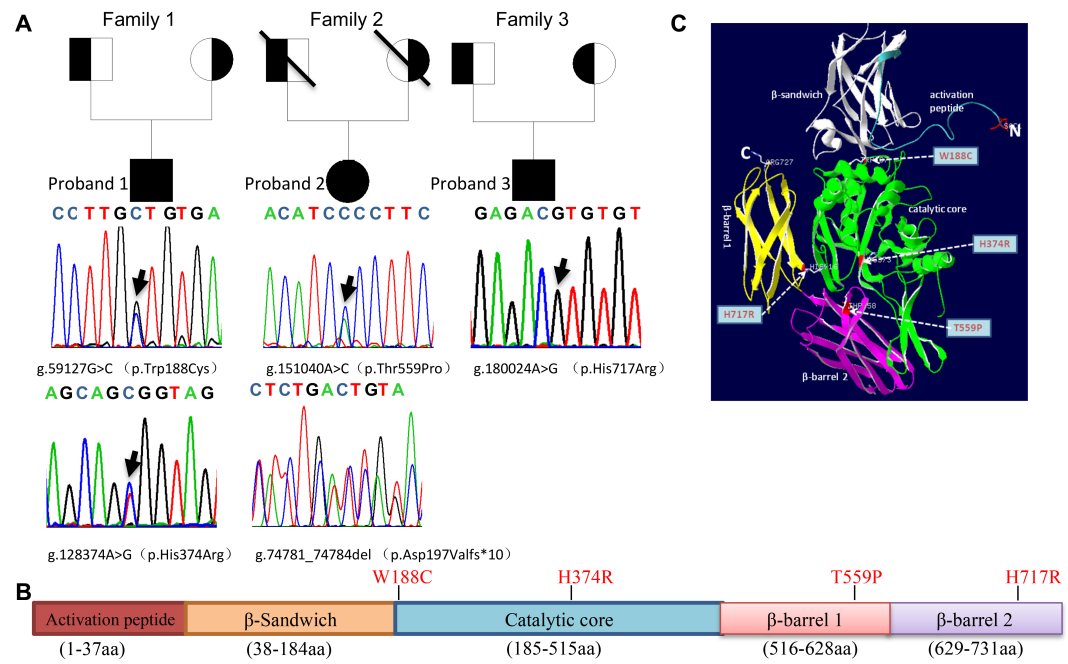

FIGURE 3

A

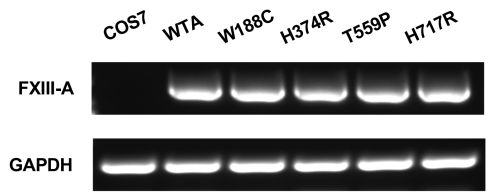

C

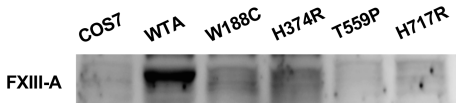

E

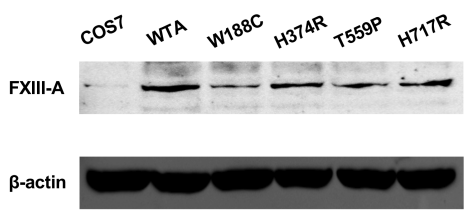

B

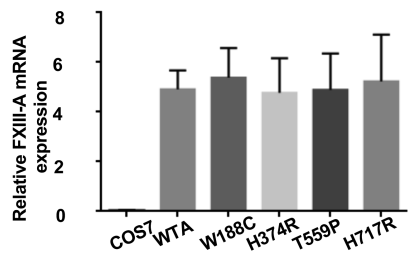

D

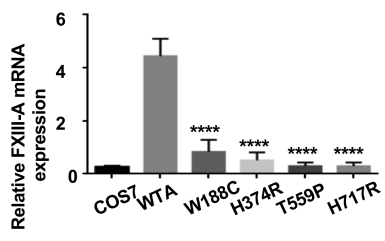

F

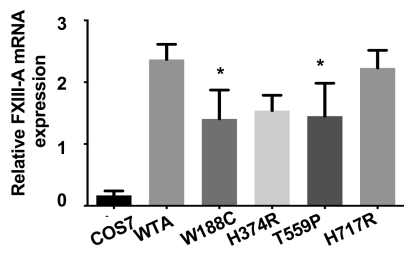


FIGURE 4

A

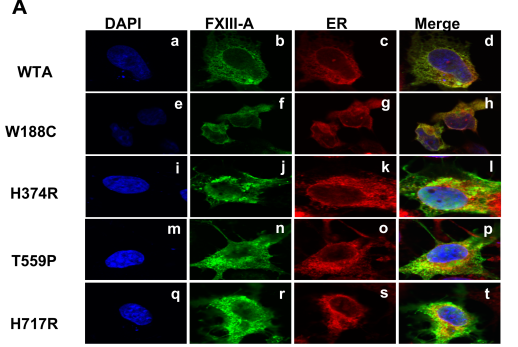

B WTA

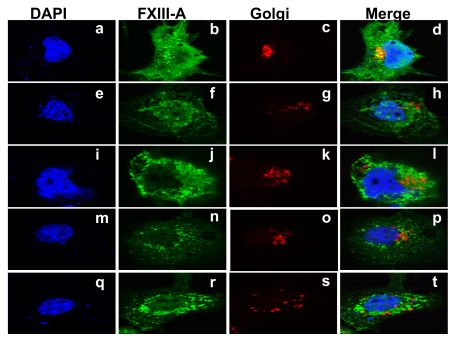

